PHYSICAL REVIEW D 82, 093003 (2010)

\title{
Theory of neutrino oscillations with entanglement
}

\author{
Boris Kayser* and Joachim Kopp ${ }^{\dagger}$ \\ Theoretical Physics Department, Fermilab, P.O. Box 500, Batavia, Illinois 60510, USA \\ R. G. Hamish Robertson \\ Department of Physics and Center for Experimental Nuclear Physics and Astrophysics, University of Washington, \\ Seattle, Washington 98195, USA \\ Petr Vogel ${ }^{\S}$ \\ Kellogg Radiation Laboratory and Physics Department, Caltech, Pasadena, California 91125, USA
}

(Received 6 July 2010; published 3 November 2010)

\begin{abstract}
We show that, despite appearances, a theoretical approach to neutrino oscillation in which the neutrino and its interaction partners are entangled yields the standard result for the neutrino oscillation wavelength. We also shed some light on the question of why plane-wave approaches to the neutrino oscillation problem can yield the correct oscillation wavelength even though they do not explicitly account for the localization of the neutrino source and the detector.
\end{abstract}

DOI: 10.1103/PhysRevD.82.093003

PACS numbers: 14.60.Pq, 03.65.Ud

\section{INTRODUCTION}

Is the standard expression [1] for the wavelength of neutrino oscillation correct? Does this expression depend correctly on the underlying neutrino mass splittings, so that the splittings that have been inferred from data using this expression are right?

The quantum-mechanical physics of neutrino oscillation has proved to be quite subtle, and has been analyzed in a variety of ways over the years. There have been treatments based on plane waves [1-4], on neutrino wave packets [5-9], and on quantum field theory [10-14]. (A thorough summary has been given recently by Akhmedov and Smirnov [15].) Most of these treatments have yielded the standard expression for the probability of oscillation, but the correct way to derive this expression is still occasionally disputed. The analysis presented in the present work, based on plane waves and on an assumed entanglement between the oscillating neutrino and a recoil particle, finds an oscillation wavelength that appears to be very different from the usual one, but turns out to be physically equivalent to it.

In this paper, we recap the recent treatment of oscillation using entangled plane waves. We express a number of concerns about such an analysis, and about plane-wave treatments in general. However, we also comment on why plane-wave treatments, while not strictly correct and consistent, nevertheless often yield the correct oscillation wavelength. We demonstrate that, in spite of concerns, and in spite of the fact that the oscillation wavelength found by considering entangled plane waves appears to be markedly different from the standard one, this wavelength, properly

\footnotetext{
*boris@fnal.gov

†jopp@fnal.gov

rghr@uw.edu

\$pxv@caltech.edu
}

understood, is physically completely equivalent to the usual one, and yields the same neutrino mass splittings when applied to data. This result is the main point of this paper.

\section{PLANE-WAVE DESCRIPTION WITH ENTANGLEMENT}

In this section, we present a derivation of the oscillation wavelength, focusing on neutrinos produced in two-body decays of the form $P \rightarrow \nu+R$, in which a parent $P$ decays into a neutrino $\nu$ and a recoil $R$. In an effort to avoid apparent paradoxes that can arise in standard plane-wave treatments of neutrino oscillations, we assume that the neutrino and its recoil form a two-body entangled state. The role of entanglement in neutrino and neutral kaon oscillations has been considered by others [16-22]. In the analysis presented here, the oscillation wavelength that emerges corresponds to the separation of the neutrino and its associated recoiling particle at a common time in the parent rest frame. This wavelength is not the usual wavelength that would be observed in an experiment (because the recoiling particle is not usually observed), but we show that the two wavelengths are physically equivalent and lead to the same neutrino survival or appearance probability. ${ }^{1}$

A neutrino of flavor $\alpha$ is a linear combination of mass eigenstates $\nu_{i}$ with masses $m_{i}$,

$$
\left|\nu_{\alpha}\right\rangle=\sum_{i} U_{\alpha i}^{*}\left|\nu_{i}\right\rangle
$$

\footnotetext{
${ }^{1}$ This section incorporates arguments presented previously in unpublished form by one of us (R.G.H.R.) [23]. A revised version of that work [24] takes into account insights explained in the present paper.
} 
where the $U_{\alpha i}$ are elements of the Maki-Nakagawa-SakataPontecorvo mixing matrix [1]. In the approach that assumes entanglement, energy-momentum conservation requires that the energy and momentum of the recoil in the decay $P \rightarrow \nu+R$ depend on which $\nu_{i}$ was actually emitted. In the rest frame of the parent, $p_{R}=-p_{\nu}$, where $p_{R}$ and $p_{\nu}$ are the momenta of the recoil and neutrino, respectively. In terms of the coordinates in that frame, the two-particle wave function may be written as

$$
\begin{aligned}
& |R \nu ; x, X\rangle \sim \sum_{i} U_{\alpha i}^{*}\left|R_{i}\left(p_{P, i}, X\right)\right\rangle\left|\nu_{i}\left(p_{\nu, i} x\right)\right\rangle \\
& \quad=\sum_{i} U_{\alpha i}^{*}|R\rangle\left|\nu_{i}\right\rangle e^{-i E_{R, i} t-i p_{\nu, i} X} e^{-i E_{\nu, i} t+i p_{\nu, i} x} \\
& \quad=|R\rangle e^{-i E_{a} t} \sum_{i} U_{\alpha i}^{*}\left|\nu_{i}\right\rangle e^{i p_{\nu, i} D} .
\end{aligned}
$$

Here, $|R\rangle$ describes the internal degrees of freedom of the recoil, and $\left|\nu_{i}\right\rangle$ simply identifies one of the three neutrino mass eigenstates. $E_{\nu, i}$ and $E_{R, i}$ are the energies of the neutrino and recoil, respectively, and $t$ is the time. The $P$-rest-frame distance between the neutrino and the recoil is $D \equiv x-X$, where the coordinates $x$ and $X$ are the positions of the neutrino and recoil, respectively. The energy $E_{a}=E_{\nu, i}+E_{R, i}=m_{P}$ in the third line of Eq. (2) is just the mass of the parent $P$. The index $i$ on $p_{\nu, i}, E_{\nu, i}$, and $E_{R, i}$ indicates that these quantities all depend on $i$, while $E_{a}$, as well as $t, x$, and $X$, do not. [To describe a neutrino oscillation experiment with a spatial resolution much better than the oscillation length, we have to fix $(t, x)$ and $(t, X)$ at the $P$-rest-frame coordinates of the space-time points where the neutrino and recoil, respectively, are detected. These points are defined by the experiment and therefore do not depend on $i$.] Note that the third line of Eq. (2) shows that, in the rest frame of the parent, the twoparticle system consisting of the neutrino and the recoil can be described by a one-particle wave function depending only on the relative coordinate $D$. This is analogous to the treatment of the hydrogen atom in most quantum mechanics textbooks, where the two-body wave function of the electron and the nucleus is factorized into a one-particle wave function describing the relative motion of the two particles, and a one-particle wave function describing the center of mass motion (which is trivial in the center of mass frame).

Suppose that in the laboratory frame, the parent $P$ is moving to the right along the $x$ axis with speed $\beta$. The labframe location $x^{\prime}$ of the neutrino when it is at the spacetime point $(t, x)$ is $x^{\prime}=\gamma(x+\beta t)$. The corresponding labframe location $X^{\prime}$ of the recoil at the same time $t$ in the $P$ rest frame is $X^{\prime}=\gamma(X+\beta t)$. Thus, the lab-frame distance between the neutrino and recoil at time $t$ in the $P$ rest frame is $x^{\prime}-X^{\prime}=\gamma(x-X) \equiv D^{\prime}$. Consequently, the state $|R \nu ; x, X\rangle$ of Eq. (2) may be rewritten as

$$
\left|R \nu ; D^{\prime}\right\rangle=e^{-i E_{a} t}|R\rangle \sum_{i} U_{\alpha i}^{*}\left|\nu_{i}\right\rangle e^{i \gamma^{-1} p_{\nu, i} D^{\prime}},
$$

where $p_{\nu, i}$ is the parent rest-frame momentum of the neutrino when the latter is $\nu_{i}$. The leading energy-dependent phase factor in Eq. (3) is unobservable. As a result, the interference effects of neutrino oscillations arise solely from the different momenta in the components in the final state. In the oscillation probability at lab-frame separation $D^{\prime}, P\left(\nu_{\alpha} \rightarrow \nu_{\beta}\right)=$ $\left|\left\langle R \nu_{\beta} \mid R \nu_{\alpha}\right\rangle_{D^{\prime}}\right|^{2}$, the $i-j$ interference term depends on $D^{\prime}$ through the phase factor $\exp \left[i \gamma^{-1}\left(p_{\nu, i}-p_{\nu, j}\right) D^{\prime}\right]$. Thus, the wavelength $\lambda_{D, i j}^{\prime}$ of oscillation in the recoil-neutrino separation $D^{\prime}$ is determined by

$$
\gamma^{-1}\left(p_{\nu, i}-p_{\nu, j}\right) \lambda_{D, i j}^{\prime}=2 \pi .
$$

Now, to leading order in $\Delta m_{i j}^{2}=m_{i}^{2}-m_{j}^{2}$,

$$
p_{\nu, i}-p_{\nu, j}=-\Delta m_{i j}^{2} \frac{1}{2 m_{P}} \frac{m_{P}^{2}+m_{R}^{2}}{m_{P}^{2}-m_{R}^{2}},
$$

where $m_{R}$ is the mass of the recoil. Thus, apart from an irrelevant sign,

$$
\lambda_{D, i j}^{\prime} \simeq \frac{4 \pi \gamma m_{P}}{\Delta m_{i j}^{2}} \frac{m_{P}^{2}-m_{R}^{2}}{m_{P}^{2}+m_{R}^{2}} .
$$

To compare this result with the standard expression for the wavelength of neutrino oscillation, it is useful to rewrite it in terms of the "neutrino beam energy" $E_{0}^{\prime}$, defined as the energy that massless neutrinos would have in the laboratory. Since $E_{0}^{\prime}=\gamma(1+\beta)\left(m_{P}^{2}-m_{R}^{2}\right) / 2 m_{P}$, and the speed $\beta_{R}$ of the recoil in the $P$ rest frame obeys $1+\beta_{R}=2 m_{P}^{2} /\left(m_{P}^{2}+m_{R}^{2}\right)$, we have

$$
\lambda_{D, i j}^{\prime}=\frac{4 \pi E_{0}^{\prime}}{\Delta m_{i j}^{2}} \frac{1+\beta_{R}}{1+\beta} .
$$

Equations (6) and (7) define a wavelength in laboratory coordinates for the separation $D^{\prime}$ between the neutrino and the recoil. In the standard expression [1] for neutrino oscillation, the survival probability oscillates as a function of the lab-frame distance $L^{\prime}$ between the neutrino source and the detector with a wavelength $\lambda_{L, i j}^{\prime}$ given by

$$
\lambda_{L, i j}^{\prime}=\frac{4 \pi E_{0}^{\prime}}{\Delta m_{i j}^{2}} .
$$

We will show in the next section that these two expressions, Eqs. (7) and (8), in fact give equivalent results for the laboratory wavelength of neutrino oscillations measured in the standard fashion by detecting neutrinos at a known distance from a source region.

A comment is in order about the role that the entanglement between the neutrino and the recoil plays for the oscillation phenomenology. In particular, one may wonder if the oscillation pattern is modified if the recoil undergoes an interaction that breaks the entanglement long before the neutrino is detected. In fact, in a typical experiment, the recoil will interact with the matter that makes up the neutrino source very soon after it has been produced. 
If it is unstable, it might also decay very rapidly. However, it is easy to see that such interaction or decay cannot change the neutrino oscillation phenomenology. First, the order in which the recoil interaction or decay and the neutrino detection occur depends on the Lorentz frame in which we are working. The neutrino flavor transition probabilities, on the other hand, are Lorentz invariant, so they cannot depend on the time ordering of these processes. In a more formal way, the same conclusion can be reached by using the quantum amplitude approach to particle mixing probabilities developed in [25]. This approach, which takes the entanglement between the neutrino and the recoil into account and confirms the validity of Eq. (2), yields the joint probability for the recoil to interact (or decay) at one spacetime point, and the neutrino to produce a charged lepton of a given flavor $\alpha$ at another space-time point. To determine the consequences of this approach for neutrino oscillations, one must integrate this joint probability over all possible interaction points of the recoil. But it turns out that the joint probability depends on the recoil interaction point $(T, X)$ only through a phase factor $e^{-i E_{R, i} T-i p_{\nu, i} X}$, analogous to the recoil phase factor we have encountered in the second line of Eq. (2). It is easy to show that this factor is independent of which neutrino mass eigenstate has been emitted together with the recoil $[22,26]$. Thus, it is an overall phase that does not influence the quantum interference between neutrino mass eigenstates that leads to neutrino oscillations. Hence, the oscillation pattern is independent of where or how soon the recoil interacts or decays, destroying its entanglement with the neutrino.

\section{PHYSICAL EQUIVALENCE OF WAVELENGTHS}

Since the wavelengths $\lambda_{D, i j}^{\prime}$ and $\lambda_{L, i j}^{\prime}$ in Eqs. (7) and (8) describe oscillations in two different variables, $D^{\prime}$ and $L^{\prime}$, let us see how these variables are related. We take the decay $P \rightarrow \nu+R$ to occur at the space-time point $(t, x)=$ $\left(t^{\prime}, x^{\prime}\right)=(0,0)$. Suppose that the neutrino is then detected at a subsequent time $t$ in the $P$ rest-frame. Then, in that frame, if the neutrino's speed is $\beta_{\nu}$, it will have traveled a distance $L=\beta_{\nu} t$ to its point of detection. The $P$ restframe separation $D$ between the neutrino and the recoil when the neutrino is detected will be $D=\beta_{\nu} t+\beta_{R} t$. Thus, since the neutrino is ultrarelativistic, with $\beta_{\nu} \simeq 1$,

$$
\frac{D}{L}=1+\beta_{R}
$$

Now, in the laboratory frame, the distance $L^{\prime}$ between the neutrino source (i.e., the location of $P$ when the decay $P \rightarrow \nu+R$ occurred) and the point of detection is $L^{\prime}=$ $\gamma(L+\beta t) \simeq \gamma(1+\beta) L$, where we have once again used $\beta_{\nu} \simeq 1$. Furthermore, as we have already seen, at the neutrino detection time $t$ in the $P$ rest frame, the laboratory-frame distance $D^{\prime}$ between the neutrino and the recoil is related to its $P$ rest-frame counterpart $D$ by
$D^{\prime}=x^{\prime}-X^{\prime}=\gamma(x-X)=\gamma D$. Thus, the variables $D^{\prime}$ and $L^{\prime}$ to which the wavelengths $\lambda_{D, i j}^{\prime}$ and $\lambda_{L, i j}^{\prime}$ refer are related by

$$
\frac{D^{\prime}}{L^{\prime}}=\frac{\gamma D}{\gamma(1+\beta) L}=\frac{1+\beta_{R}}{1+\beta} .
$$

By comparison, from Eqs. (7) and (8),

$$
\frac{\lambda_{D, i j}^{\prime}}{\lambda_{L, i j}^{\prime}}=\frac{1+\beta_{R}}{1+\beta} .
$$

That is, the ratio between the "new" wavelength $\lambda_{D, i j}^{\prime}$ of oscillation in $D^{\prime}$ and the standard wavelength $\lambda_{L, i j}^{\prime}$ of oscillation in $L^{\prime}$ is exactly the same as that between $D^{\prime}$ and $L^{\prime}$.

Thus, although the new $\lambda_{D, i j}^{\prime}$ of Eq. (7) has emerged from an approach that entails entanglement, while the standard $\lambda_{L, i j}^{\prime}$ of Eq. (8) has come from analyses that generally do not, these two wavelengths differ only because they refer to two different, alternative distance variables. Thus, they are physically equivalent. Properly used to fit given oscillation data, they would yield precisely the same neutrino squared-mass splitting $\Delta m_{i j}^{2}$.

In practice, of course, an analysis of experimental data using Eq. (7) instead of the usual Eq. (8) would require knowledge of the coordinates of both the neutrino and the recoil at the same time $t$ in the parent rest frame. The required coordinates of the recoil would be extremely difficult to obtain. However, it is not necessary to actually detect the recoil if the space-time trajectory and energy of the parent particle are known - as they would be, for instance, in an electron-capture beta-beam neutrino experiment. In that case, energy-momentum conservation could be used to infer the trajectory of the recoil from the kinematics of the parent and the measured coordinates of the neutrino.

\section{WHY IS THE SAME $\Delta m^{2}$ OBTAINED?}

So long as the standard approaches and the approach that invokes entanglement are all valid ways of deriving the neutrino oscillation wavelength, the wavelengths derived by these approaches obviously must be correct and physically equivalent. In the previous section, we have demonstrated the physical equivalence for a particular approach that invokes entanglement and that obtains the wavelength for oscillation in the separation $D^{\prime}$ between the neutrino and its recoiling partner at a given time $t$ in their common parent's rest frame. But this must be a more general result. Consider a "gedanken" neutrino oscillation experiment, with some relevant properly defined distance $D^{\prime \prime}$ between the neutrino and its recoiling partners, and with $D^{\prime \prime}$ proportional to the usual source-to-detector distance $L^{\prime}$. Then, the ratio between the wavelengths of oscillation in $D^{\prime \prime}$ and $L^{\prime}$ will be $D^{\prime \prime} / L^{\prime}$, so that these two wavelengths will be physically equivalent. 
Another way of understanding intuitively why the standard expression for the oscillation length is obtained even when the neutrino and the recoiling particle are considered as an entangled state is the following: Let us choose a frame in which the component of the recoil that is entangled with neutrino mass eigenstate $\nu_{1}$ of mass $m_{1}$ is at rest; i.e. $p_{R, 1}^{\prime \prime}=0$. We denote kinematic quantities in this frame by a double prime. From simple kinematic arguments, it follows that

$$
\begin{gathered}
p_{R, j}^{\prime \prime}=\frac{\Delta m_{j 1}^{2} m_{R}}{m_{P}^{2}-m_{R}^{2}}+\cdots \\
E_{R, j}^{\prime \prime}=m_{R}+\cdots,
\end{gathered}
$$

where "..." denotes terms that are at least fourth order in the neutrino masses, divided by combinations of $m_{R}$ and $m_{P}$. The complex phase of the $j$ th component of the entangled state has the form

$$
-E_{R, j}^{\prime \prime} t^{\prime \prime}+p_{R, j}^{\prime \prime} X^{\prime \prime}-E_{\nu, j}^{\prime \prime} t^{\prime \prime}+p_{\nu, j}^{\prime \prime} x^{\prime \prime} .
$$

We see that, at leading order in $\Delta m_{j 1}^{2}$, the first term is a constant that does not contribute to the phase differences relevant to neutrino oscillations and can therefore be omitted. We will now show that the second term can be neglected as well. Since we are using a plane-wave approach here, it is not immediately obvious what $X^{\prime \prime}$ is; after all, plane waves are delocalized over space. We will argue below that it is reasonable to impose the relations $x^{\prime \prime}=v_{\nu}^{\prime \prime} t^{\prime \prime}$ and $X^{\prime \prime}=v_{R}^{\prime \prime} t^{\prime \prime}$ by hand, where it is justified to take $v_{\nu}^{\prime \prime}$ and $v_{R}^{\prime \prime}$ to be the averages of the group velocities associated with the individual components of the neutrino and the recoil, respectively. In the frame where $p_{R, 1}^{\prime \prime}=0$, all $v_{R j}^{\prime \prime}$ are proportional to $\Delta m_{j 1}^{2}$, so that no matter how exactly the average $v_{R}^{\prime \prime}$ is defined, $p_{R, j}^{\prime \prime} X^{\prime \prime}$ is second order in $\Delta m_{j 1}^{2}$ and therefore negligible. Consequently, the phase reduces to the standard expression

$$
-E_{\nu, j}^{\prime \prime} t^{\prime \prime}+p_{\nu, j}^{\prime \prime} x^{\prime \prime}
$$

that does not depend on the properties of the recoil. Because of Lorentz invariance, the phase must therefore be the standard one in any frame.

\section{PLANE WAVES VS WAVE PACKETS}

It is clear that, due to the Heisenberg uncertainty principle, neutrinos produced and detected in localized regions of space-time must have a nonzero spread in energy and momentum. Many authors have argued that, to take this spread into account, a fully consistent theoretical treatment of neutrino oscillations requires wave packets $[5-7,10,12,14]$. Other authors have argued that wave packets are unnecessary [27].

Indeed, in most neutrino oscillation experiments, wave packet effects, such as wave packet separation due to different group velocities [5], are negligible, and simplified plane-wave approaches can correctly predict experimental results. The deeper reason for this can be understood if we note that the coordinate space representation of a typical Gaussian neutrino wave packet,

$$
\psi_{j}(\mathbf{x}, t) \propto e^{-i E_{j 0} t+i \mathbf{p}_{j 0} \mathbf{x}} \exp \left[\frac{\left(\mathbf{x}-\mathbf{v}_{j} t\right)^{2}}{4 \sigma_{x}^{2}}\right]
$$

is simply a plane wave, multiplied with an enveloping Gaussian. Here, the index $j$ distinguishes different neutrino mass eigenstates, $\mathbf{p}_{j 0}$ and $E_{j 0}$ are the average momentum around which the wave packet's momentum distribution is peaked and the associated energy; $\mathbf{v}_{j}=\mathbf{p}_{j 0} / E_{j 0}$ is the group velocity, and $\sigma_{x}$ is the width of the wave packet in coordinate space. The neutrino oscillation probability depends on the phase differences between wave packets associated with different mass eigenstates $j$. We observe that, at each fixed space-time point, these phase differences depend only on $E_{j 0}$ and $\mathbf{p}_{j 0}{ }^{2}$ They do not depend on $\sigma_{x}$, so they are independent of the precise shape of the wave packets, and, in particular, remain unchanged in the limit $\sigma_{x} \rightarrow \infty$ corresponding to plane waves. They do, however, vary over space and time. In the full wave packet picture, the enveloping Gaussian ensures that only space-time points along the trajectory $\mathbf{x} \sim \mathbf{v} t$, and located at the detector site, $\mathbf{x} \simeq \mathbf{L}$, contribute to the oscillation probability. ${ }^{3}$ In a plane-wave approach, this has to be ensured by imposing $\mathbf{x}=\mathbf{v} t$ and $\mathbf{x}=\mathbf{L}$ by hand.

Another argument not to invoke wave packets is the observation by Kiers, Nussinov, and Weiss [8] that a continuous flux of neutrino wave packets with identical momentum distributions cannot be distinguished from an ensemble of plane-wave neutrinos, whose individual momenta follow the same momentum distribution. In fact, the density matrices describing the two ensembles are identical.

Even though the above arguments show that plane-wave approaches to neutrino oscillations can be justified, there are inconsistencies with the quantum field theoretic (QFT) formalism, where the production and detection processes are explicitly included in the calculation and the neutrino is treated as an internal line in a Feynman diagram (see Fig. 1). Since energy and momentum are exactly conserved at the production and detection vertices, the intermediate neutrino can only be an on-shell energy-momentum eigenstate (as it has to be if it propagates over macroscopic distances) if its interaction partners-the external lines of the Feynman diagram - are also energy-momentum eigenstates. However, it is clear that the external energies and

\footnotetext{
${ }^{2}$ Note that interference can only occur between wave packet components located at the same point in space-time.

${ }^{3}$ Here, $\mathbf{v}$ should be understood as an average of the individual $\mathbf{v}_{j}$, which is a valid concept as long as the wave packets have not yet separated. Also note that $|\mathbf{v}| \simeq c$, with small corrections to this relation being a negligible second-order effect in the small neutrino masses.
} 


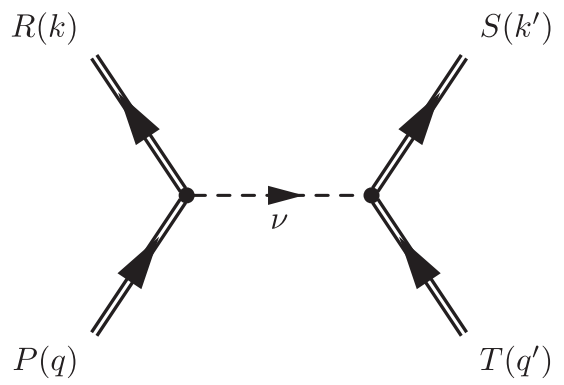

FIG. 1. The Feynman diagram of neutrino oscillations. $P$ and $R$ are the parent particle and the recoil, respectively, while $T$ is the target atom at the detector and $S$ denotes the outgoing "signal" particles.

momenta in this case cannot be the same for diagrams involving different neutrino mass eigenstates. Since in quantum mechanics interference is only possible between amplitudes for processes that describe different paths leading to the same final state, this means that no oscillations are possible [12].

A similar argument can also be invoked to show that, in the QFT treatment, entanglement between the neutrino and its interaction partners cannot persist asymptotically for $t \rightarrow \infty$. Such asymptotic entanglement would lead to different external states in diagrams involving different neutrino mass eigenstates, so interference between these diagrams would be impossible. If we take the point of view that initially the neutrino is entangled with at least some of its interaction partners, we are led to the conclusion that these particles must be disentangled by further interactions for oscillations to take place. (This has been noticed previously in Ref. [17].) Disentanglement is achieved when the particles interact with their environment. These interactions localize them and thus introduce momentum uncertainties that are usually large enough to allow external particle states entangled with different neutrino mass eigenstates to interfere in spite of their different energies and momenta.

With this in mind, one can justify a plane-wave approach even in QFT. The procedure is to compute the amplitudes corresponding to different neutrino mass eigenstates and then sum them coherently (before squaring), keeping in mind that such coherent summation is only justified if the final state particles undergo further interactions that introduce energy and momentum uncertainties larger than the energy and momentum differences between the different entangled states.

If one wishes to avoid such reasoning, one can also directly incorporate the effect of the localizing secondary interactions into the Feynman diagram computation by treating the external particles as wave packets with appropriate energy and momentum spreads. Different neutrino mass eigenstates will then simply couple to different (though usually overlapping) portions of the external wave packets' momentum distributions. Feynman diagrams involving different neutrino mass eigenstates can thus have identical external states, so that interference and therefore neutrino oscillations emerge.

\section{CONCLUSIONS}

For the reasons we have explained, one must be cautious when following a plane-wave approach to neutrino oscillation, whether or not the approach incorporates entanglement. A plane-wave approach does not include the neutrino source and detector localizations that are physically essential if oscillation with distance is to be observed. Consequently, such an approach cannot be used to discover all the implications of these localizations. However, the explicit inclusion of localization effects, which is automatic in wave packet treatments, can be replaced for some purposes by physical reasoning in a plane-wave approach. Indeed, the oscillation wavelength derived from a plane-wave analysis, with or without entanglement, can be perfectly correct. In particular, as we have shown, the wavelength that emerges from the analysis presented in this paper, which does invoke entanglement, is physically equivalent to the (correct) wavelength obtained by standard wave packet or plane-wave treatments. Thus, one may continue to have confidence in the neutrino mass-squared splittings $\Delta m^{2}$ that have been deduced by applying the standard wavelength expression to data.

\section{ACKNOWLEDGMENTS}

We would like to thank S. Parke for organizing a very fruitful discussion session about entanglement in neutrino oscillations at Fermilab. We are also indebted to E. Akhmedov, J. Conrad, G. Garvey, T. Goldman, M. Goodman, B. Keister, J. Lowe, M. Messier, M. Shaevitz, A. Smirnov, R. Volkas, W. Winter, and L. Wolfenstein for inspiring and useful discussions. The work of P. V. was partially supported by the U.S. Department of Energy under Contract No. DE-FG02-88ER40397. The work of R.G.H.R. was supported by the U.S. Department of Energy under Contract No. DE-FG02-97ER41020. Fermilab is operated by Fermi Research Alliance, LLC under Contract No. DE-AC02-07CH11359 with the U.S. Department of Energy. 
[1] C. Amsler et al. (Particle Data Group), Phys. Lett. B 667, 1 (2008).

[2] A. Mann and H. Primakoff, Phys. Rev. D 15, 655 (1977).

[3] S. M. Bilenky and B. Pontecorvo, Phys. Rep. 41, 225 (1978).

[4] H. J. Lipkin, Phys. Lett. B 642, 366 (2006).

[5] S. Nussinov, Phys. Lett. 63B, 201 (1976).

[6] B. Kayser, Phys. Rev. D 24, 110 (1981).

[7] C. Giunti, C. W. Kim, and U. W. Lee, Phys. Rev. D 44, 3635 (1991).

[8] K. Kiers, S. Nussinov, and N. Weiss, Phys. Rev. D 53, 537 (1996).

[9] C. Giunti and C. W. Kim, Fundamentals of Neutrino Physics and Astrophysics (Oxford University Press, Oxford, UK, 2007).

[10] W. Grimus and P. Stockinger, Phys. Rev. D 54, 3414 (1996).

[11] W. Grimus, P. Stockinger, and S. Mohanty, Phys. Rev. D 59, 013011 (1998).

[12] M. Beuthe, Phys. Rep. 375, 105 (2003).

[13] C. Giunti, J. High Energy Phys. 11 (2002) 017.
[14] E. K. Akhmedov and J. Kopp, J. High Energy Phys. 04 (2010) 008 .

[15] E. K. Akhmedov and A. Y. Smirnov, Phys. At. Nucl. 72, 1363 (2009).

[16] J. Goldman, Mod. Phys. Lett. A 25, 479 (2010).

[17] A. G. Cohen, S. L. Glashow, and Z. Ligeti, Phys. Lett. B 678, 191 (2009).

[18] A. Dolgov, A. Morozov, L. Okun, and M. Schepkin, Nucl. Phys. B502, 3 (1997).

[19] H. Burkhardt, J. Lowe, G. Stephenson Jr., and J. Goldman, Phys. Lett. B 566, 137 (2003).

[20] H. Burkhardt, J. Lowe, G. Stephenson Jr., and J. Goldman, Phys. Rev. D 59, 054018 (1999).

[21] J. Lowe et al., Phys. Lett. B 384, 288 (1996).

[22] E. K. Akhmedov and A. Y. Smirnov, arXiv:1008.2077.

[23] R. G. H. Robertson, arXiv:1004.1847v1.

[24] R. G. H. Robertson, arXiv:1004.1847v2.

[25] B. Kayser and L. Stodolsky, Phys. Lett. B 359, 343 (1995).

[26] A. Y. Smirnov (private communication).

[27] L. Stodolsky, Phys. Rev. D 58, 036006 (1998). 